\title{
BIM NO PLANEJAMENTO DE EMPREENDIMENTOS COM SISTEMAS DE PAINÉIS PRÉ-FABRICADOS: UMA ALTERNATIVA PARA OBRAS DE HABITAÇÃO SOCIAL
}

\section{BIM IN THE PLANNING PROCESS OF PROJECTS WITH PREFABRICATED PANEL SYSTEMS: AN ALTERNATIVE TO SOCIAL HOUSING CONSTRUCTION}

\author{
Pedro Henrique Pinto Bezerra ${ }^{1}$ \\ Universidade Federal do Paraná, Curitiba, PR, Brasil, bezerra.pedro92@gmail.com
}

Adriana de Paula Lacerda Santos ${ }^{2}$

Universidade Federal do Paraná, Curitiba, PR, Brasil, adrianapls@ufpr.br

Sergio Scheer 3

Universidade Federal do Paraná, Curitiba, PR, Brasil, scheer@ufpr.br

\section{Resumo}

A pré-fabricação na construção ou construção off-site é apontada por pesquisadores como uma alternativa de industrializar o processo construtivo e atingir melhores níveis de desempenho. A utilização dos sistemas de painéis pré-fabricados, por sua vez, é considerada uma opção mais flexível e simples dentre os diversos tipos de construção off-site existentes. Estes sistemas também são apontados como ideais para a construção de habitações de interesse social, em razão do seu alto desempenho sustentável e execução simples. Além disso, devido ao déficit habitacional verificado em países menos desenvolvidos, novos métodos construtivos que tornem a construção de habitações populares mais rápida e barata são necessários. Portanto, o presente artigo busca investigar, por meio do metodologia de Revisão Sistemática da Literatura, como aplicação de Building Information Modeling (BIM) pode auxiliar no planejamento de empreendimentos com painéis pré-fabricados, de modo a chamar atenção para o potencial de uso dessas tecnologias como forma de suprir a demanda por moradia em países emergentes e subdesenvolvidos.

Palavras-chave: Painéis pré-fabricados. Habitação social. Building Information Modeling.

\begin{abstract}
Prefabrication in construction, or off-site construction, is pointed out by researchers as an alternative to industrialize the construction process and achieve better levels of performance. The use of prefabricated panel systems, in turn, is considered a more flexible and simpler option among the various types of off-site construction. These systems are also indicated as ideal for the construction of social housing, because of its high sustainable performance and easy execution. Moreover, due to the housing shortage verified in less developed countries, new construction methods that make the construction of social housing faster and cheaper are necessary. Therefore, this paper aims to investigate, through the methodology of Systematic Review of Literature, how the application of Building Information Modeling (BIM) can aid in the planning process of projects with prefabricated panels, in order to highlight the potential use of these technologies as a way of addressing the demand for housing in emerging and underdeveloped countries.
\end{abstract}

Keywords: Prefabricated panel. Social housing. Building Information Modeling.

How to cite this article:

BEZERRA, Pedro Henrique Pinto; SANTOS, Adriana de Paula Lacerda; SCHEER, Sergio. BIM no planejamento de empreendimentos com sistemas de painéis pré-fabricados: uma alternativa para obras de habitação social. PARC Pesquisa em Arquitetura e Construção, Campinas, SP, v. 9, n. 3, p. 192-203, set. 2018. ISSN 1980-6809. Disponível em:

<https://periodicos.sbu.unicamp.br/ojs/index.php/parc/article/view/8650218>. Acesso em: 21 maio 2018.

doi:https://doi.org/10.20396/parc.v9i3.8650218. 


\section{Introdução}

A pré-fabricação/pré-montagem externa, também chamada de construção off-site (fora do canteiro) é proposta por pesquisadores e profissionais de construção como uma abordagem efetiva para industrializar os empreendimentos de construção e alcançar níveis de desempenho mais altos, como nas indústrias de produtos de consumo e automóveis (SAID; CHALASANI; LOGAN, 2017).

Dentre os diversos tipos de métodos de pré-fabricação, os sistemas de painéis pré-fabricados são considerados pela indústria como um método de construção viável devido à sua flexibilidade, podendo ser aplicado tanto na construção de fachadas, quando para paredes interiores. Nestes sistemas, o comprimento das paredes é dividido em painéis, com larguras que devem atender a restrições de transporte (SAID; CHALASANI; LOGAN, 2017).

Os painéis são produzidos em fábricas e, posteriormente, enviados à obra para instalação. Este processo, em comparação com o método convencional de construção, minimiza o desperdício no processo construtivo e aumenta a eficiência. No entanto, o sucesso de tais projetos depende da programação precisa para a fabricação, envio e instalação dos painéis, pois falhas na entrega ou superprodução nas fábricas podem resultar em atrasos nos projetos e custos de armazenamento (LIU et al., 2014).

Assim, investigações sobre formas de aprimorar o processo de projeto e planejamento de obras com esses sistemas são oportunas para promovê-los como uma alternativa para a construção de habitações de interesse social, de modo a suprir a grande demanda por moradia verificada em países subdesenvolvidos (ARAÚJO et al, 2016a).

\section{Vantagens e desafios da utilização de sistemas de painéis pré-fabricados em obras de habitação social}

Para Vivan, Paliari e Novaes (2010), os sistemas de painéis pré-fabricados representam uma importante contribuição alternativa para a industrialização do setor de edificações, em contraposição ao sistema tradicional, que apesar de apresentar processos e tecnologias mais acessíveis aos profissionais, é caracterizado, no caso das habitações unifamiliares, por grandes desperdícios de materiais, pela lentidão no processo de produção e por ser mais susceptível ao surgimento de manifestações patológicas durante o uso do produto.

A produção dos painéis em um ambiente controlado pode ser especialmente benéfica quando se tenta evitar problemas causados, por exemplo, por condições climáticas adversas. A pré-fabricação permite que as construções sejam montadas e revestidas de forma mais rápida, reduzindo os custos significativamente em comparação aos métodos tradicionais (TAM, 2013).

Ainda segundo Vivan, Paliari e Novaes (2010), no sistema tradicional vários componentes da construção são produzidos com bases artesanais, gerando produtos que possuem características como imprecisão dimensional, qualidade variável, baixo controle tecnológico etc. Surgem também, durante a produção, atividades de fluxo que convergem para o desperdício de mão-de-obra e materiais, resultando em prejuízos e improdutividade. Assim, na construção de conjuntos residenciais, tendo em vista nos números do déficit habitacional brasileiro, isso se torna impraticável.

Araujo et al. (2016a) aborda o uso de painéis préfabricados em madeira, sistema conhecido como Woodframe, e seu potencial para a construção de habitações de interesse social. Para os autores, a adoção desta tecnologia por parte dos governos de países da América Latina permitiria a expansão do mercado para esse tipo de sistema construtivo. As empresas da cadeia de produção de madeira, ou seja, os fabricantes e fornecedores de matérias-primas também seriam beneficiados. Ainda é destacada a vantagem econômica da utilização dos painéis, que ocasionam uma redução de quase $50 \%$ dos custos de produção de uma habitação nos padrões do programa "Minha Casa Minha Vida", do governo brasileiro.

Ainda sobre o uso de Woodframe para habitação social, Araujo et al. (2016b) afirma que esse sistema é o mais conveniente para fins de construção residencial, especialmente devido a critérios como segurança, prevenção de incêndio, eficiência energética, qualidade e estética. Assim, a construção de habitações com painéis pré-fabricados permite cada vez mais que os construtores ofereçam aos consumidores casas mais baratas do que aquelas construídas por métodos convencionais, sem sacrificar a qualidade da casa ou o apelo estético.

\section{Uso de Building Information Modeling em construções pré-fabricadas}

A modelagem de informações de construção (BIM) é uma ferramenta importante para a indústria de pré-fabricação devido à sua capacidade de fornecer um protótipo virtual preciso dos componentes físicos do edifício antes da produção em fábrica. Além do processo de fabricação e construção dos componentes de construção, o BIM também desempenha um papel importante durante a construção e os estágios operacionais de um edifício (LIU et al., 2014).

Com a utilização de sistemas BIM, é possível planejar a sequência de construção de forma mais eficiente e prática. Quando comparados à modelagem tradicional, baseada em 
desenhos 2D que incluem apenas informações geométricas da edificação, sem nenhum outro tipo de informação ou conhecimento sobre a construção, os sistemas BIM oferecem informações aprimoradas sobre cada elemento, assim possibilitando vários tipos de análises (LIU et al., 2014).

Segundo El Debs e Ferreira (2012), os sistemas BIM permitem que os projetos sejam desenvolvidos colaborativamente, aumentando a capacidade de correção de erros e a resolução de incompatibilidades. Essa melhoria na qualidade do projeto contribui para a utilização de elementos pré-fabricados, já que estes não podem ser alterados na obra.

No estudo feito por Battacherjee, Pishdad-Bozorgi e Ganapathy (2016), onde os autores analisam o processo de projeto de um empreendimento executado com o sistema de painéis pré-fabricados, o uso coordenado de BIM foi crucial na fase pré-operacional, tornando possível a realização de análise da construtibilidade, desenvolvimento de desenhos para fabricação compatíveis e estimativa dos custos. Alinhando todos os modelos em um ambiente virtual, o time de projeto pôde identificar erros de dimensões, determinar quantidades de materiais para instalação dos painéis, coordenar detalhes de impermeabilização e rastrear os pedidos.

Assim, o uso de sistemas BIM oferece uma alternativa de aprimoramento do processo construtivo com painéis préfabricados, abrindo caminho para a maior aplicação deste método na construção de habitações de interesse social.

Assim, o presente trabalho buscará investigar especificamente as contribuições recentes sobre a utilização de BIM no planejamento de obras residenciais com painéis pré-fabricados e avaliar o potencial de aplicação da tecnologia em obras de habitações populares em países emergentes e subdesenvolvidos. Para isso, foi adotado o método de Revisão Sistemática da Literatura.

\section{Método}

Como forma de avaliar o tema proposto, foi adotado o método de Revisão Sistemática da Literatura (RSL). Tratase de um tipo de investigação que disponibiliza um resumo das evidências relacionadas a uma estratégia de intervenção específica, mediante a aplicação de métodos explícitos e sistematizados de busca, apreciação crítica e síntese da informação selecionada (SAMPAIO; MANCINI, 2007).

O principal alcance da RSL é integrar a informação existente sobre uma temática específica, através do agrupamento e análise dos resultados procedentes de estudos primários realizados em locais e momentos diferentes por grupos de pesquisa independentes, permitindo a geração de evidência científica sobre temática abordada (MUÑ̃Z et al., 2002).

As revisões sistemáticas são particularmente úteis para integrar as informações de um conjunto de estudos realizados separadamente sobre determinada intervenção, que podem apresentar resultados conflitantes e/ou coincidentes, bem como identificar temas que necessitam de evidência, auxiliando na orientação para investigações futuras (SAMPAIO; MANCINI, 2007).

No início do processo de revisão sistemática, é proposta uma pergunta que a pesquisa buscará responder, que consiste, geralmente, em avaliar a relação de causalidade existente entre dois fatores, obedecendo uma sequência de passos a serem percorridos. Em seguida, três etapas devem ser cumpridas: definir o objetivo da revisão, identificar a literatura e selecionar os estudos possíveis de serem incluídos. Essas etapas preliminares são importantes, uma vez que auxiliam os pesquisadores a adequar a pergunta norteadora da revisão com base na informação disponível sobre o tema de interesse (SAMPAIO; MANCINI, 2007; OLSEN, 1995 apud MUÑOZ et al., 2002).

Desse modo, a questão principal que a presente revisão buscará responder é: "Como a tecnologia BIM pode ser usada para auxiliar no planejamento de obras de habitação social que utilizam sistemas de painéis pré-fabricados como método construtivo?".

Após a formulação da pergunta norteadora da pesquisa, prosseguiu-se com o processo de identificar a literatura existente e avaliar quais artigos seriam úteis para a análise pretendida. Foi, então, elaborado o que Sampaio e Mancini (2007) chamam protocolo de pesquisa, que consiste em definir como os estudos serão encontrados, os critérios de exclusão, os desfechos de interesse e avaliar a qualidade dos estudos. Nessa etapa, devem ser escolhidos termos ou palavras-chave, seguidos de estratégias de busca, definição das bases de dados e outras fontes de informação.

Foram escolhidos dois repositórios de publicações online: ScienceDirect e ASCE Library. Considerou-se também utilizar a plataforma Scopus, porém, para os termos de busca escolhidos na presente RSL, não foi encontrada uma quantidade relevante de publicações nesta base. Desse modo, foram também escolhidos como fontes de informação o mecanismo de busca Google Acadêmico e o portal Periódicos Capes, por estas englobarem diversas bases de dados em suas buscas.

Em cada das fontes selecionadas, foram feitas duas buscas utilizando os seguintes conjuntos de palavras-chave: "PANELIZED CONSTRUCTION BIM" e "PANELIZED CONSTRUCTION SOCIAL HOUSING", levando em consideração apenas publicações dos últimos 5 anos. A busca foi feita somente com termos em inglês por 
considerar-se que as publicações nacionais sobre a temática são escassas.

A partir dos resultados das buscas, foi realizada uma primeira filtragem das publicações encontradas, eliminando aquelas que apareciam mais de uma vez. Em seguida, foi feita uma segunda filtragem, dessa vez levando em consideração apenas o título das publicações, eliminando aquelas que não se relacionavam com a temática abordada.

As publicações foram, então, analisadas de forma ainda mais minuciosa, dessa vez com base nos seus resumos (abstracts), sendo possível eliminar ainda mais artigos irrelevantes para a pesquisa. Em seguida, as publicações restantes foram lidas na integra, o que ocasionou ainda mais uma filtragem, pois percebeu-se que muitos dos artigos não se encaixavam na questão principal da presente revisão (Tabela 1 e 2).

Tabela 1 - Resultados das buscas feitas nas quatro bases de dados.
\begin{tabular}{|c|c|c|c|c|}
\hline $\begin{array}{c}\text { PALAVRAS- } \\
\text { CHAVE }\end{array}$ & \multicolumn{4}{|c|}{ NÚMERO DE PUBLICAÇÕES } \\
\cline { 2 - 5 } & $\begin{array}{c}\text { Google } \\
\text { Acadêmico }\end{array}$ & $\begin{array}{c}\text { Science } \\
\text { Direct }\end{array}$ & $\begin{array}{c}\text { ASCE } \\
\text { Library }\end{array}$ & $\begin{array}{c}\text { Periódicos } \\
\text { CAPES }\end{array}$ \\
\hline $\begin{array}{c}\text { PANELIZED } \\
\text { CONSTRUCTION } \\
\text { BIM }\end{array}$ & 185 & 6 & 23 & 12 \\
\hline $\begin{array}{c}\text { PANELIZED } \\
\text { CONSTRUCTION } \\
\begin{array}{c}\text { SOCIAL } \\
\text { HOUSING }\end{array}\end{array}$ & 189 & 12 & 30 & 22 \\
\hline
\end{tabular}

Fonte: $\mathrm{O}$ autor.

Tabela 2 - Filtragem das publicações.
\begin{tabular}{|c|c|}
\hline DESCRIÇÃO & QUANT. \\
\hline TOTAL DE PUBLICAÇÕES & 479 \\
\hline PUBLICAÇÕES NÃO DUPLICADAS & 347 \\
\hline $\begin{array}{c}\text { PRIMEIRA FILTRAGEM - aderência ao tema em estudo } \\
\text { identificada no título }\end{array}$ & 135 \\
\hline $\begin{array}{c}\text { SEGUNDA FILTRAGEM - aderência ao tema em estudo } \\
\text { confirmada no resumo }\end{array}$ & 48 \\
\hline $\begin{array}{c}\text { TERCEIRA FILTRAGEM - aderência ao tema em estudo } \\
\text { confirmada no texto completo }\end{array}$ & 15 \\
\hline
\end{tabular}

Fonte: 0 autor.

Após a seleção dos 15 artigos, foi feito um processo conhecido como "bola de neve". Nesta etapa, todas as referências dos artigos escolhidos por meio das filtragens foram analisadas, a fim de verificar os estudos mais citados e avaliar se essas publicações seriam relevantes para o presente estudo. Foi possível, então, encontrar duas publicações que se adequavam ao escopo do trabalho.

A revisão resultou em 17 artigos no total, sendo a grande maioria das pesquisas feitas na América do Norte, predominantemente no Canadá. Também foram encontradas publicações na América do Sul, Europa e Oceania. A distribuição é mostrada no Gráfico 1.
Por fim, os artigos foram lidos e seus conteúdos analisados. Os dados foram extraídos de duas formas: primeiro dos artigos que tratavam do planejamento de obras com sistemas de painéis pré-fabricados, separando os artigos que aplicavam a tecnologia BIM neste processo e as publicações que abordavam outras tecnologias. Em seguida, foram analisados os artigos que tratavam da utilização de painéis pré-fabricados em obras de habitação social. Os resultados são mostrados a seguir.

Gráfico 1 - Distribuição das publicações encontradas por continente.

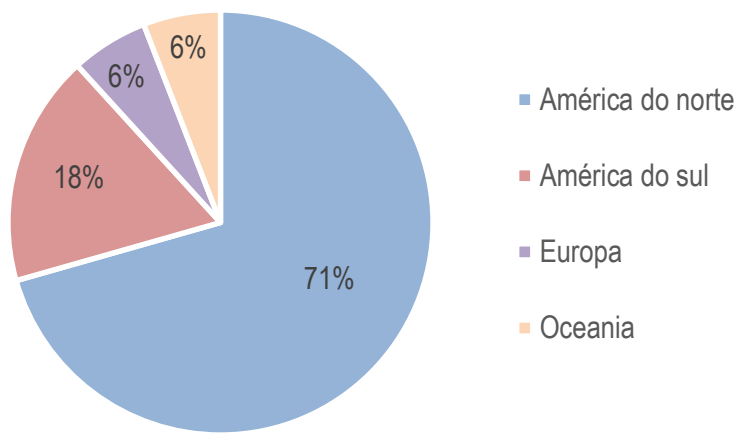

Fonte: 0 autor.

\section{Análise de conteúdo}

\section{BIM como ferramenta de apoio ao planejamento de construções com painéis pré-fabricados}

A primeira parte da leitura das publicações encontradas foi composta por 8 artigos que tratavam da aplicação de BIM tanto no planejamento de obras com painéis pré-fabricados quanto no aprimoramento do processo de produção destes elementos (Tabela 3 ).

Foram identificadas diferentes estratégias de melhoria para a fabricação de painéis pré-fabricados para construção. Em Hamdan et al. (2015), os autores chamam atenção para a necessidade de uma gestão eficaz dos estoques, ou seja, para a escolha do volume apropriado de produção dos painéis, de modo que o ritmo de fabricação destes componentes esteja em consonância com a execução das obras. Para isso, os autores usam as informações contidas em um modelo BIM para alimentar um software de simulação e testar as sequências de produção, sendo possível escolher um padrão adequado para a fabricação dos painéis. Em Liu et al. (2015), verificou-se uma abordagem semelhante, integrando a tecnologia BIM com o conceito de simulação de eventos discretos ${ }^{1}$, tornando possível avaliar a linha de produção dos painéis e planejar, por exemplo, a ordem de fabricação considerando cada tipo de painel (interior ou exterior) e verificar a sequência mais rápida. 
Por sua vez, Salama, Salah e Moselhi (2016) também analisam o processo de produção dos painéis, propondo um método de planejamento baseado no conceito de Linear Scheduling Method $^{2}$, considerando a incerteza associada à produtividade das equipes envolvidas no processo de manufatura por meio do Método da Corrente
Crítica $^{3}$. O sistema proposto depende da tecnologia BIM para automatizar a aquisição de dados, por meio dos quais torna-se possível calcular as durações das atividades de fabricação, incorporando as incertezas inerentes ao processo produtivo.

Tabela 3 - BIM tanto no planejamento de obras com painéis pré-fabricados e no aprimoramento do processo de produção destes elementos

\begin{tabular}{|c|c|c|c|}
\hline AUTOR & FOCO & $\begin{array}{l}\text { FERRAMENTAS } \\
\text { UTILIZADAS }\end{array}$ & RESULTADOS \\
\hline $\begin{array}{l}\text { Hamdan et al. } \\
\qquad(2015)\end{array}$ & Gestão de estoques & $\begin{array}{l}\text { Modelo BIM, simulação } \\
\text { contínua e simulação de } \\
\text { eventos discretos }\end{array}$ & $\begin{array}{l}\text { O sistema criado permite a importação de diferentes tipos de } \\
\text { modelos de edificações e melhora a visualização dos processos, } \\
\text { possibilitando um maior controle da produção dos painéis. Apresenta } \\
\text { limitações quanto à interface gráfica e é pouco flexível quando à } \\
\text { leitura das informações dos modelos BIM. }\end{array}$ \\
\hline Liu et al. (2014) & $\begin{array}{l}\text { Geração automática de } \\
\text { planejamento de obras }\end{array}$ & $\begin{array}{c}\text { Autodesk Revit, MS Access e } \\
\text { MS Project }\end{array}$ & $\begin{array}{c}\text { É desenvolvido um sistema de planejamento automático de } \\
\text { atividades, baseado no Autodesk Revit. É concluído que o sistema } \\
\text { criado poderá ajudar a planejar as atividades de maneira mais } \\
\text { eficiente e reduzirá os erros humanos na programação da } \\
\text { construção com painéis pré-fabricados. }\end{array}$ \\
\hline Liu et al. (2015) & $\begin{array}{l}\text { Criação de um modelo de } \\
\text { simulação baseado em BIM para } \\
\text { a linha de produção de painéis }\end{array}$ & $\begin{array}{l}\text { Autodesk Revit, } \\
\text { Symphony.NET (University of } \\
\text { Alberta) }\end{array}$ & $\begin{array}{l}\text { O modelo criado facilita a avaliação do desempenho da linha de } \\
\text { produção de painéis, permitindo o planejamento e controle da } \\
\text { produção por meio de princípios enxutos. }\end{array}$ \\
\hline $\begin{array}{l}\text { Liu, Al-Hussein e } \\
\qquad \text { Lu (2015) }\end{array}$ & $\begin{array}{l}\text { Abordagem, baseada em BIM, } \\
\text { para o planejamento de obras sob } \\
\text { restrições de recursos }\end{array}$ & $\begin{array}{l}\text { Autodesk Revit, MS Access e } \\
\text { Symphony. NET (University of } \\
\text { Alberta) }\end{array}$ & $\begin{array}{c}\text { Foi desenvolvido um protótipo de sistema de planejamento de obras } \\
\text { com painéis pré-fabricados por meio de uma extensão para } \\
\text { Autodesk Revit, capaz de produzir programações compatíveis com o } \\
\text { MS Project. O sistema é capaz de produzir as progamações } \\
\text { esperadas para construções com painéis, e auxilia de modo eficaz } \\
\text { no planejamento da montagem em canteiro, reduzindo a } \\
\text { possibilidade de erros. }\end{array}$ \\
\hline $\begin{array}{l}\text { Liu, Lu e Al- } \\
\text { Hussein. (2016) }\end{array}$ & $\begin{array}{l}\text { Proposta de uma abordagem } \\
\text { semântica baseada em ontologias } \\
\text { para gerar listas de quatitativos } \\
\text { com termos específicos do } \\
\text { sistema de painéis }\end{array}$ & Autodesk Revit & $\begin{array}{c}\text { O trabalho estabelece uma ontologia de produtos orientada à } \\
\text { construção, que complementa os modelos BIM adicionando termos } \\
\text { específicos, propriedades e inter-relações entre elementos. É } \\
\text { possível, assim, extrair quantitativos com termos específicos, } \\
\text { relativos aos componentes dos painéis, de modo a facilitar o } \\
\text { planejamento da produção. }\end{array}$ \\
\hline $\begin{array}{l}\text { Salama, Salah e } \\
\text { Moselhi (2016) }\end{array}$ & $\begin{array}{l}\text { Proposta de um método de } \\
\text { planejamento considerando } \\
\text { incertezas no processo de } \\
\text { produção dos painéis }\end{array}$ & $\begin{array}{l}\text { Software Vertex BD (BIM), } \\
\text { Linear Scheduling Method } \\
\text { (LSM) }\end{array}$ & $\begin{array}{l}\text { O método desenvolvido representa uma alternativa para o } \\
\text { planejamento de construções pré-fabricadas, sem necessidade de } \\
\text { uso de modelos de simulação. É possível planejar a produção dos } \\
\text { painéis considerando a linearidade da linha de produção e as } \\
\text { incertertas associadas aos índices de produtividade. }\end{array}$ \\
\hline $\begin{array}{l}\text { Samarasinghe et } \\
\text { al. (2015) }\end{array}$ & $\begin{array}{l}\text { Apresentação de um modelo de } \\
\text { processo de projeto para casas } \\
\text { pré-fabricadas por meio do uso de } \\
\text { diversos softwares BIM }\end{array}$ & $\begin{array}{l}\text { Autodesk Revit, IES VE, } \\
\text { FirstRate5, Autodesk Robot, } \\
\text { INSUL, Autodesk Navisworks }\end{array}$ & $\begin{array}{l}\text { Foi feita a modelagem de uma casa inteiramente pré-fabricada, } \\
\text { sendo possível realizar diversos tipos de análises por meio dos } \\
\text { softwares utilizados, escolhidos em função da interoperabilidade. } \\
\text { Concluiu-se que o modelo pode ser utilizado para diversos sistemas } \\
\text { construtivos (pré-fabricados ou não). }\end{array}$ \\
\hline $\begin{array}{l}\text { Barkokebas et } \\
\quad \text { al. (2015) }\end{array}$ & $\begin{array}{l}\text { Uso da extração automática de } \\
\text { quantitativos de modelos BIM } \\
\text { para estimativa de custos }\end{array}$ & Vico Software & $\begin{array}{c}\text { O fluxo de trabalho de uma empresa que utiliza painéis pré- } \\
\text { fabricados em suas obras foi estudado, identificando-se possíveis } \\
\text { áreas de melhoria. Com a utilização do software Vico, foi possível } \\
\text { reduzir o tempo gasto para a realização dos orçamentos em } 30 \% \text { e a } \\
\text { entrega de outros documentos relativos ao planejamento não foi } \\
\text { prejudicada. }\end{array}$ \\
\hline
\end{tabular}

Fonte: Os autores.

Também foi possível identificar estratégias de aplicação da tecnologia BIM nas fases de planejamento da construção com painéis pré-fabricados. Em Liu et al. (2014) e Liu, Al-Hussein e Lu (2015), são abordadas propostas de geração automática da programação da obra a partir das informações extraídas de modelos BIM. Samarasinghe et al. (2015) chama atenção para a necessidade de adaptação do processo de projeto em BIM para as construções pré-fabricadas, desenvolvendo uma metodologia específica para a concepção deste tipo de empreendimento.

Em Barkokebas et al. (2015) identificou-se um enfoque diferente, tratando da utilização de BIM para criação de projetos inteligentes, que sejam capazes de fornecer informações geométricas e quantitativos que possam ser compreendidos por todos os agentes envolvidos no empreendimento, permitindo uma maior agilidade na 
estimativa dos custos de produção. Liu, Lu e Al-Hussein (2016) também abordam problemas na compreensão das informações dos modelos BIM, relacionados às listas de quantitativos extraídas. Desse modo, é proposta uma abordagem semântica baseada em ontologias para a extração de quantitativos orientados à construção.

Após a análise dos estudos, foi feito um levantamento das ferramentas utilizadas nas pesquisas e resumidos os seus resultados, como forma de visualizar as vantagens do uso de BIM em construções com painéis pré-fabricados. Considerou-se importante ainda analisar algumas outras publicações que aplicam outras tecnologias no planejamento de construções com painéis pré-fabricados, a fim de avaliar a eficiência dessas outras alternativas frente aos sistemas BIM (Tabela 4).

Em Inyang et al. (2012), buscou-se avaliar os riscos ergonômicos associados à montagem de painéis préfabricados nas obras por meio de Realidade Virtual. A metodologia baseou-se na criação de um banco dados, integrado a um software de simulação, permitindo a análise da sequência de execução adequada. Um modelo de Realidade Virtual é, então, criado com base na simulação, sendo possível avaliar aspectos ergonômicos e de produtividade.

Said, Chalasani e Logan (2017) apontam a necessidade de pesquisa por novas abordagens para aprimorar a customização do projeto e da fabricação de painéis para construção. Assim, os autores desenvolvem um modelo de plataforma ${ }^{4}$ de painéis exteriores otimizado, de modo a analisar um possível equilíbrio entre a maximização da variedade dos painéis quanto ao seu desenho (solido, com janelas, com portas, etc) e o aumento da eficiência na fabricação. Altaf et al. (2015) exploram a integração entre um sistema RFID (Radio Frequency Identification System) e a simulação de eventos discretos de modo a monitorar o estado da produção de painéis em um dado momento no modelo de simulação, permitindo acompanhamento em tempo real. Por fim, em Altaf, Al-Hussein e Yu (2014), o conceito de simulação de eventos de discretos também é utilizado em conjunto com um algoritmo de otimização de modo a mitigar as dificuldades no processo de produção de painéis em Woodframe.

\begin{tabular}{|c|c|c|c|}
\hline AUTOR & FOCO & FERRAMENTAS UTILIZADAS & RESULTADOS \\
\hline $\begin{array}{l}\text { Inyang et al. } \\
\text { (2012) }\end{array}$ & $\begin{array}{l}\text { Aplicação de Realidade Virtual } \\
\text { como forma de avaliar a os riscos } \\
\text { ergonomicos existentes durante a } \\
\text { construção com painéis pré- } \\
\text { fabricados }\end{array}$ & $\begin{array}{l}\text { MS Access, Symphony.NET } \\
\text { (University of Alberta), 3DS } \\
\text { Max, ErgoCheck }\end{array}$ & $\begin{array}{l}\text { Baseado na análise das posturas e movimentações dos operários } \\
\text { verificadas no modelo de Realidade Virtual, foi possível propor um } \\
\text { novo layout para o local de trabalho, diminuindo o tempo de } \\
\text { execução dos painéis e a ocorrência de movimentações prejudiciais } \\
\text { aos operários }\end{array}$ \\
\hline $\begin{array}{l}\text { Said, Chalasani } \\
\text { e Logan (2017) }\end{array}$ & $\begin{array}{l}\text { Desenvolvimento de um modelo } \\
\text { de plataforma de otimização para } \\
\text { fabricação de painéis }\end{array}$ & $\begin{array}{l}\text { Desenvolvimento de algoritmos } \\
\text { computacionais (simulações) }\end{array}$ & $\begin{array}{l}\text { Foi desenvolvido um modelo de plataforma de otimização que ajuda } \\
\text { a diminuir o custo total de fabricação e a manter a variabilidade do } \\
\text { projeto dos painéis. É proposto ainda que o sistema seja aprimorado } \\
\text { com o uso de Building Information Modeling, de modo a melhorar a } \\
\text { visualização dos processos. }\end{array}$ \\
\hline Altaf et al. (2015) & $\begin{array}{l}\text { Apresentação de uma } \\
\text { metodologia para integrar um } \\
\text { modelo de simulação da } \\
\text { fabricação dos painéis com um } \\
\text { sistema de aquisição de dados } \\
\text { automatizado } \\
\end{array}$ & $\begin{array}{c}\text { MS Access, Symphony.NET } \\
\text { (University of Alberta), sistema } \\
\text { RFID }\end{array}$ & $\begin{array}{l}\text { Com o uso de um sistema RFID para coleta de dados em tempo } \\
\text { real, foi possível criar um modelo de simulação que ajuda no } \\
\text { controle da produção, tornando-o mais dinâmico e menos } \\
\text { susceptível ao erro humano. O novo sistema gera informações } \\
\text { automaticamente e permite que as decisões sobre diferentes opções } \\
\text { de produção sejam tomadas mais rapidamente. }\end{array}$ \\
\hline $\begin{array}{l}\text { Altaf, Al-Hussein } \\
\text { e Yu (2014) }\end{array}$ & $\begin{array}{l}\text { Integração de simulação de } \\
\text { eventos discretos com um } \\
\text { algoritmo de otimização de modo } \\
\text { a automatizar o processo de } \\
\text { sequenciamento dos painéis }\end{array}$ & $\begin{array}{l}\text { Symphony.NET (University of } \\
\text { Alberta), algoritmo de } \\
\text { otimização por núvem de } \\
\text { partículas }\end{array}$ & $\begin{array}{l}\text { Foi desenvolvido um modelo de simulação integrado ao um } \\
\text { algoritmo de otimização por meio do uso de um banco de dados } \\
\text { contendo informaçães sobre os painéis. É possível então usar a } \\
\text { simulação para analisar melhorias na produtividade. }\end{array}$ \\
\hline
\end{tabular}

Fonte: 0 autor.

A partir da leitura dos artigos, foi possível avaliar as contribuições recentes sobre as tecnologias e ferramentas computacionais que estão sendo utilizadas de modo a aprimorar o processo de produção de painéis préfabricados e sua execução no canteiro de obras. Em seguida, serão verificadas as publicações encontradas na Revisão Sistemática da Literatura sobre o uso específico de painéis pré-fabricados em obras de habitação social, para que no item 3 deste trabalho, seja possível relacionar os resultados e responder à questão principal definida para a pesquisa.

\section{Aplicação de painéis pré-fabricados em obras de habitação social}

A segunda parte da leitura das publicações encontradas foi feita nos artigos que tratavam da aplicação de painéis préfabricados para habitação social (Tabela 5). O foco desta etapa foi na identificação dos benefícios que este método construtivo pode trazer para as obras de habitação social em países em desenvolvimento.

Salzer et al. (2016) destacam a necessidade de um desenvolvimento mais sustentável e inclusivo para a 
habitação social em países subdesenvolvidos. Os autores estudam o potencial de criação de um novo sistema de painéis pré-fabricados baseado na utilização de bambu, no contexto das Filipinas, país onde este material é abundante. Tam (2013), por sua vez, analisa o potencial de aplicação de construções pré-fabricadas no mercado asiático, sobretudo no Vietnam, que, em decorrência de um crescimento econômico recente, tem presenciado uma demanda cada vez maior por habitações.

Em Araujo et al. (2016a) e Araujo et al. (2016b), destacam-se as vantagens da construção em madeira, sobretudo com o sistema construtivo em Woodframe. São apontadas suas vantagens, como racionalização de materiais, baixo consumo de água, limpeza dos canteiros de obra e rápida execução. Os autores também chamam atenção para o déficit habitacional pelo qual os países latino-americanos têm passado nos últimos anos, o que elimina a possibilidade de aquisição da casa própria pelas populações mais pobres. Com estes fatos em vista, é apontada a necessidade de pesquisas relacionadas à construção com painéis pré-fabricados. Por fim, Vivan, Paliari e Novaes (2010) evidenciam que o processo de industrialização facilita a produção seriada de residências para conjuntos habitacionais, em razão da repetitividade presente no processo construtivo, apontando a utilização do sistema de painéis em Light Steel Framing ou Steelframe como solução viável para suprir a demanda por habitação social.

Desse modo, como feito anteriormente, foram organizados os métodos de pesquisa e os resultados das publicações desta parte da revisão (Tabela 5).

Tabela 5 - Aplicação de painéis pré-fabricados para habitação social

\begin{tabular}{|c|c|c|c|}
\hline AUTOR & FOCO & METODOLOGIA & RESULTADOS \\
\hline Salzer et al. (2016) & $\begin{array}{l}\text { Uso do bambu como alternativa } \\
\text { mais sustentável para a } \\
\text { construção de habitações } \\
\text { sociais em países } \\
\text { subdesenvolvidos }\end{array}$ & Entrevistas, estudos de caso & $\begin{array}{c}\text { Os autores utilizaram } 15 \text { critérios para avaliar a sustentabilidade do } \\
\text { bambu como alternativa para a construção de habitação social. Foi } \\
\text { proposto um roteiro para o desenvolvimento de um sistema construtivo } \\
\text { pré-fabricado utilizando o bambu, chamando a atenção para a } \\
\text { necessidade de pesquisa sobre alternativas mais sustentáveis para } \\
\text { habitação social nos grandes centros urbanos de países } \\
\text { subdesenvolvidos. }\end{array}$ \\
\hline Tam (2013) & $\begin{array}{l}\text { Análise das oportunidades de } \\
\text { uso de pré-fabricação na } \\
\text { construção de casas no } \\
\text { Vietnam }\end{array}$ & $\begin{array}{l}\text { Avaliação do potencial de } \\
\text { expansão da indústria de } \\
\text { construção pré-fabricada } \\
\text { canadense para o cenário } \\
\text { vietnamita }\end{array}$ & $\begin{array}{c}\text { É avaliado que o Vietnam é um país de intenso crescimento } \\
\text { econômico atualmente e cuja população cresce cada vez mais, } \\
\text { aumentando a demanda por habitações. São feitas recomentações } \\
\text { quanto ao tipo de sistema pré-fabricado que se adequaria melhor à } \\
\text { realidade do país e quanto à necessidade de adaptação às normas } \\
\text { construtivas vietnamitas. }\end{array}$ \\
\hline $\begin{array}{l}\text { Araujo et al. } \\
\quad(2016 a)\end{array}$ & $\begin{array}{l}\text { Construção pré-fabricada em } \\
\text { madeira como uma alternativa } \\
\text { mais sustentável }\end{array}$ & $\begin{array}{c}\text { Levantamento bibliográfico do } \\
\text { estado da arte sobre } \\
\text { construções em madeira }\end{array}$ & $\begin{array}{c}\text { É verificado que a industrialização e o aumento do volume de madeira } \\
\text { na construção tem grande potencial para substituir os materiais } \\
\text { tradicionais, que estão ficando ultrapassados e possuem um baixo } \\
\text { desempenho sustentável. Nesse sentido, o uso de sistemas pré- } \\
\text { fabricados em madeira oferece uma alternativa eficaz, especialmente } \\
\text { para países com déficit habitacional }\end{array}$ \\
\hline $\begin{array}{l}\text { Araujo et al. } \\
\quad(2016 b)\end{array}$ & $\begin{array}{l}\text { Uso do sistema construtivo em } \\
\text { Woodframe na construção de } \\
\text { habitações na América Latina }\end{array}$ & $\begin{array}{l}\text { Levantamento bibliográfico } \\
\text { sobre o sistema construtivo } \\
\text { em Woodframe e as } \\
\text { vantagens de sua utilização }\end{array}$ & $\begin{array}{c}\text { A partir do levantamento realizado, os autores concluem que a maior } \\
\text { barreira para a maior utilização do sistema de Woodframe é a cultura } \\
\text { do mercado e o desconhecimento das pessoas. É chamado atenção } \\
\text { para participação de pesquisadores e universidades na pesquisa } \\
\text { voltada ao aprimoramento desse sistema, como forma de promover } \\
\text { sua disseminação no mercado. }\end{array}$ \\
\hline $\begin{array}{l}\text { Vivan. Paliari e } \\
\text { Novaes (2010) }\end{array}$ & $\begin{array}{l}\text { Vantagem produtiva do sistema } \\
\text { construtivo em Steelframe }\end{array}$ & $\begin{array}{l}\text { Levantamento bibliográfico } \\
\text { sobre construção enxuta e } \\
\text { racionalização construtiva e } \\
\text { descrição do sistema } \\
\text { construtivo em Steelframe }\end{array}$ & $\begin{array}{l}\text { É apontado que existe a necessidade de incorporar princípios da } \\
\text { construção enxuta na indústria de construção, de modo a promover a } \\
\text { racionalização construtiva. Destaca-se o pontencial do sistema em } \\
\text { Steelframe para contemplar esses princípios, com grande capacidade } \\
\text { de suprir a demanda por moradias existente atualmente }\end{array}$ \\
\hline
\end{tabular}

Fonte: $\mathrm{O}$ autor.

\section{Discussão}

Neste item, será feita uma reflexão sobre o tema abordado, com base nos resultados da Revisão Sistemática da Literatura realizada, a fim de responder à pergunta principal definida para a pesquisa, que consiste em investigar como os sistemas BIM podem contribuir para o planejamento de obras de habitação social que utilizem sistemas de painéis pré-fabricados como método construtivo.

A análise dos resultados da revisão foi dividida em duas partes, primeiro tratando dos trabalhos sobre o planejamento de obras com sistemas de painéis pré- 
fabricados, e depois das pesquisas sobre a aplicação deste método construtivo em obras de habitação social.

Foi possível observar na primeira parte da revisão que o processo construtivo com painéis pré-fabricados requer uma adaptação das práticas de planejamento tradicionais, por envolver, segundo Liu et al. (2014), dois processos que abrangem diferentes ênfases em relação às melhorias de produtividade: gestão do processo de manufatura, durante a fase de produção dos painéis nas fábricas, e planejamento e controle das operações de montagem desses elementos, durante a execução dos empreendimentos.

Assim, os resultados da revisão mostram que os sistemas BIM oferecem uma alternativa eficaz para gerir esses dois processos, pois foram encontradas publicações que abordavam a utilização dessa tecnologia em ambos. $\mathrm{Na}$ produção de painéis, é usada principalmente integrando as informações extraídas dos modelos BIM a ferramentas de simulação para analisar sequencias de produção, organizar estações de trabalho, e mesmo definir o ritmo de fabricação de acordo com o andamento da obra (HAMDAN et al., 2015; LIU et al., 2015a; SALAMA et $a l ., 2016)$. Na fase de planejamento dos empreendimentos, também aliada a mecanismos de simulação, pode ser usada para fornecer uma base de dados para gerar programações da obra automaticamente (LIU et al., 2014; LIU et al., 2015b), para extrair quantitativos de modo a auxiliar na execução das obras (LIU et al., 2016), para realizar análises de conforto, sustentabilidade e compatibilização (SAMARASINGUE et al., 2015) e para automatizar a realização de orçamentos (BARKOKEBAS et al., 2015).

Foram identificadas ainda algumas outras publicações que tratavam sobre o planejamento de obras com painéis préfabricados, mas que aplicavam outras tecnologias, como Realidade Virtual (INYANG et al., 2016), algoritmos computacionais de otimização (ALTAF; AL-HUSSEIN, YU, 2014; SAID; CHALASANI; LOGAN, 2017) e sistemas RFID (ALTAF et al., 2015). No entanto, foi possível observar que em todos esses trabalhos, a tecnologia BIM poderia ter sido aplicada de alguma forma, sem que a complexidade dos processos estudados aumentasse.

Em Inyang et al. (2012), é criado um modelo de Realidade Virtual para analisar os riscos ergonômicos associados à montagem dos painéis. O modelo é desenvolvido com base em uma simulação das atividades, criada a partir de um banco de dados. A aplicação de BIM nesse processo poderia ocorrer de duas maneiras: servindo como base de dados para simulação ou mesmo como a própria ferramenta de visualização dos riscos ergonômicos, eliminando a necessidade do modelo de Realidade Virtual. Contudo, a tecnologia BIM só poderia ser usada nesse sentido em fases pré-operacionais, considerando os riscos ainda na fase de projeto (RAJENDRAN et al., 2011). Essa substituição seria oportuna pelo fato dos sistemas BIM já serem conhecidos no setor de construção, enquanto a criação de modelos de Realidade Virtual é menos comum no mercado.

Em Altaf, Al-Hussein e Yu (2014), é usado um algoritmo de otimização para simular de sequenciamento da produção dos painéis, usando um banco de dados com informações sobre a geometria dos painéis como base. Nesse caso, a tecnologia BIM também poderia ser usada para alimentar o banco de dados, como evidenciado no trabalho de Liu et al. (2015a). No estudo feito por Altaf et al. (2015), o sistema idealizado também poderia usar modelos BIM como repositório de dados para simulação. Já em Said, Chalasani e Logan (2017), os próprios autores concluem que a utilização de BIM poderia melhorar a ferramenta criada no estudo, aprimorando a capacidade de visualização e extração de dados.

Na segunda parte da revisão, foi possível observar uma ênfase na utilização de matérias-primas mais sustentáveis para a produção de painéis voltados à habitação social. No trabalho de Salzer et al. (2016), foi possível observar que a utilização do bambu, material abundante nas Filipinas, onde o estudo foi feito, constitui uma alternativa interessante para a construção de habitações em países menos desenvolvidos, que em geral enfrentam problemas de déficit habitacional. Em Araujo et al. (2016a) e Araujo et al. (2016b), foram observadas várias vantagens da construção por meio de painéis em madeira (Woodframe), destacando-se a facilidade de montagem, curto tempo de execução, baixo consumo de água, flexibilidade de projeto, limpeza e organização nos canteiros de obra.

Vivan, Paliari e Novaes (2010) chamam atenção para a aplicabilidade dos painéis pré-fabricados em obras de conjuntos habitacionais, em razão da natureza repetitiva desses tipos de obras. Além disso, em todos esses estudos, é destacada a necessidade de busca por novos métodos construtivos que tornem a construção de habitações sociais mais barata e mais eficiente, em razão da escassez habitacional que muitos países vivem atualmente. Portanto, a utilização de painéis pré-fabricados, como evidenciado em Tam (2013), tem grande potencial para suprir essa demanda, por ser um sistema construtivo sustentável, facilmente executável e de fabricação simples.

A partir da análise dos trabalhos, foi possível observar ainda algumas lacunas que precisam ser tratadas em futuros trabalhos. Hamdan et al. (2015) e Barkokebas et al. (2015) chamam atenção para a criação de sistemas capazes de interpretar informações de qualquer modelo BIM, sem que haja necessidade de modelagens padronizadas, além de uma demanda por maior difusão dessa tecnologia no mercado, pois muitos profissionais ainda resistem a adotá-la. 
As maiores lacunas, no entanto, encontram-se nas publicações que tratam da geração automática do planejamento das obras ou da produção de painéis. Salama, Salah e Moselhi (2016) e Liu et al. (2015) destacam a necessidade de criar métodos capazes de identificar gargalos e possíveis atrasos na linha de produção e de considerar tempos de operação baseados em dados reais. Por sua vez, são encontradas limitações nos estudos de Liu et al. (2014) e Liu, Al-Hussein e Lu (2015) relacionadas a utilização de dados sobre recursos e produtividade baseados na experiência ao invés de dados históricos, além de outros fatores como clima e espaço de trabalho não serem considerados. Liu, Al-Hussein e $\mathrm{Lu}$ (2015) ainda apontam que parte do sistema criado no estudo ainda demanda trabalho manual.

A maioria das publicações analisadas utiliza uma abordagem de planejamento baseada nos componentes pré-fabricados, ou seja, os autores programam a obra em relação a cada elemento a ser instalado e não às atividades, como normalmente é feito. Assim, uma possível agenda de pesquisa futura seria a criação de métodos que considerem todas atividades da obra, não apenas a instalação dos componentes pré-fabricados. Além disso, o uso de BIM para a geração automática do planejamento é outra abordagem a ser aprimorada. Algoritmos metaheurísticos como a otimização por nuvem de partículas (Particle Swarm Optimization) e Redes Neurais (Artificial Neural Networks), são ferramentas que tem potencial para auxiliar nesse processo, podendo também ser abordados em futuras pesquisas (ALTAF et al., 2014; LIU et al., 2015; LIU; ALHUSSEIN; LU, 2015).

Assim, com base em todos os estudos analisados, é possível perceber claramente que a utilização de BIM no planejamento de obras com painéis pré-fabricados poderia ser bastante oportuna na construção de habitações para populações mais pobres. Com o déficit habitacional existente, alternativas que tornem o processo de projeto e construção de habitações populares mais rápido são muito necessárias, o que faz dos sistemas BIM uma opção boa para tornar os processos de fabricação mais eficazes e a permitir uma execução mais ágil para esses tipos de empreendimentos.

\section{Conclusão}

O presente trabalho procurou investigar a forma com que a tecnologia BIM pode ser aplicada para aprimorar o processo produtivo de obras que utilizem sistemas de painéis pré-fabricados como método construtivo. Além disso, também foi avaliada a aplicação destes sistemas em obras de habitação social, chamando atenção para a necessidade de novos processos construtivos que possam ajudar a suprir o déficit habitacional vivido em países mais pobres.
Desse modo, a metodologia utilizada foi a de Revisão Sistemática da Literatura, de modo a responder o seguinte questionamento norteador da pesquisa: "Como a tecnologia BIM pode ser usada para auxiliar no planejamento de obras de habitação social que utilizam sistemas de painéis pré-fabricados como método construtivo?".

Foram utilizados dois repositórios de publicações online para a realização da pesquisa: ScienceDirect e ASCE Library; acrescidos de buscas pelo Google Acadêmico e no Portal de Periódicos Capes. Como palavras-chave para a busca foram utilizados os termos "PANELIZED CONSTRUTION \& BIM" e "PANELIZED CONSTRUTION \& SOCIAL HOUSING", encontrando um total de 479 publicações. Os resultados da pesquisa foram, então, filtrados quatro vezes levando em consideração, primeiramente, as publicações duplicadas. A segunda filtragem foi feita analisando os títulos que fugiam do escopo da pesquisa, seguida por mais duas filtragens baseadas nos resumos dos artigos (abstracts) e conteúdo completo, respectivamente. Foi então realizado um processo conhecido como "bola de neve", onde foram analisadas as referências das publicações filtradas, de modo a encontrar outros trabalhos relevantes para a pesquisa. $\mathrm{O}$ resultado final da revisão contou com 17 publicações.

Procedeu-se, em seguida, a identificação do foco de cada artigo, como forma de analisar separadamente cada aspecto necessário para responder à questão norteadora da revisão. Foram identificados 8 artigos que tratavam diretamente da utilização de BIM no planejamento de construções com painéis pré-fabricados, 4 publicações que também falavam do planejamento desse tipo de empreendimento, mas com a aplicação de outras tecnologias, e outros cinco trabalhos que analisavam a utilização de painéis pré-fabricados em obras de habitação social.

Todos os artigos foram analisados em detalhe, sendo possível identificar o uso de BIM tanto no aprimoramento do processo de fabricação dos painéis, quanto no planejamento de obras que os usem. Foi destacado que, nos trabalhos encontrados sobre o planejamento destas obras sem o uso de BIM, a tecnologia poderia ter sido utilizada em alguma etapa sem o que a complexidade dos processos estudados aumentasse, mostrando que os sistemas BIM oferecem uma alternativa de aplicação relevante e com potencial de fácil incorporação. Por sua vez, nos trabalhos sobre aplicação de painéis préfabricados em habitação social, foi possível identificar diversas vantagens do uso deste método construtivo na construção de residências para populações menos favorecidas, como ganhos em desempenho ambiental, 
redução de custos de produção e simplificação e agilidade de execução.

Foi possível ainda indentificar algumas lacunas presentes nos estudos, que poderiam servir de base para pesquisadores em futuras pesquisas. Para a fabricação dos painéis, é ainda necessário criar sistemas que possam extrair dados de qualquer modelo BIM, sem que seja necessário realizar modelagens adaptadas a um sistema em particular. A geração automática de cronogramas e planos para as obras também requer aprimoramento, pois em muitos trabalhos identificou-se a criação de planejamentos pouco precisos. Sugere-se, em pesquisas futuras, que ferramentas computacionais de otimização como algoritmos metaheurísticos (ALTAF; AL-HUSSEIN; YU, 2014; LIU; AL-HUSSEIN; LU, 2015) sejam explorados, de modo a aprimorar as programações criadas.

Por fim, pode-se concluir que, devido aos problemas de déficit habitacional que muitos países vivem atualmente, a busca por soluções que tornem a construção de habitações populares mais rápida e barata é demandada, e que a tecnologia BIM poderia ser aliada à adoção do sistema construtivo de painéis pré-fabricados para que essa demanda seja suprida, fornecendo habitações sustentáveis e de qualidade para as populações mais pobres.

\section{Notas}

(1) Tipo de simulação capaz de reproduzir processo de construção reais, com base em programação computadorizada e análises estatísticas. É capaz de fornecer aos gerentes de construção uma visão sobre os sistemas construtivos, avaliando vários cenários possíveis. Assim, configura-se como uma ferramenta poderosa para realizar análises abrangentes, melhorando a produtividade de processos de produção marcados pela repetitividade (LIU et al, 2015a).

(2) O Linear Scheduling Method (LSM) é um avanço em relação à tradicional técnica de Linha de Balanceamento, geralmente utilizada no planejamento de processos repetitivos. O LSM uma matemática semelhante à Linha de Balanceamento, porém permite a representação de atividade não repetitivas e atípicas em processos construtivos complexos (SALAMA et al., 2016).

(3) O Método da Corrente Crítica é uma técnica de planejamento onde é identificada a cadeia de atividades mais longa, que determina a duração total do projeto, sendo chamada de cadeia de suprimentos crítica. Geralmente é necessário em projetos com limitado número de recursos, de modo que as atividades da cadeia crítica são dependentes pois utilizam os mesmos recursos, devendo necessariamente ser executadas sequencialmente (SALAMA et al., 2016).

(4) Plataforma é conceituado como uma família de itens que compõe a arquitetura do produto. No caso dos painéis préfabricados, essa família seria representada pelos perfis de madeira ou aço que formam a estrutura do painel, placas de vedação, isolamento, etc. A plataforma é criada como uma forma de padronizar os componentes do produto, permitindo que os mesmos elementos sejam utilizados na fabricação de produtos diferentes (paredes externas, internas, com janelas, com portas, etc) (SAID et al., 2017).

\section{Referências}

ALTAF, Mohammed Sadiq et al. Online simulation modeling of prefabricated wall panel production using RFID system. In: WINTER SIMULATION CONFERENCE (WSC), 2015, Huntington Beach, Estados Unidos. Proceedings... Nova Jersey: IEEE, 2015. 3379-3390. doi:https://doi.org/10.1109/WSC.2015.7408499.

ALTAF, Mohammed Sadiq; AL-HUSSEIN, Mohamed; YU, Haitao. Wood-frame wall panel sequencing based on discrete-event simulation and particle swarm optimization. In: INTERNATIONAL SYMPOSIUM ON AUTOMATION AND ROBOTICS IN CONSTRUCTION AND MINING (ISARC), 31., 2014, Sydney, Austrália. Proceedings... [S.I. : s.n.]. 254-261. doi: https://doi.org/10.22260/ISARC2014/0034.

ARAUJO, Victor A. et al. Wooden residential buildings-A sustainable approach. Bulletin of the Transilvania University of Brasov. Forestry, Wood Industry, Agricultural Food Engineering. Series II, v. 9, n. 2, p. 53, 2016.

ARAUJO, Victor A. et al. Woodframe: light framing houses for developing countries. Revista de la Construcción. Journal of Construction, v. 15, n. 2, p. 78-87, 2016. doi:http://dx.doi.org/10.4067/S0718-915X2016000200008.

BARKOKEBAS, Beda et al. Coordination of cost estimation for industrialized residential projects through the use of BIM. In: INTERNATIONAL SYMPOSIUM ON AUTOMATION AND ROBOTICS IN CONSTRUCTION AND MINING (ISARC), 32., 2015, Oulu, Finlândia. Proceedings... [S.I. : s.n.]. 312-317. doi:https://doi.org/10.22260/ISARC2015/0039. 
BHATTACHARJEE, Suchismita; PISHDAD-BOZORGI, Pardis; GANAPATHY, Ram. Adoption of pre-fabrication in construction to achieve sustainability goals: an empirical study. In: CONSTRUCTION RESEARCH CONGRESS, 2016, San Juan, Porto Rico. Proceedings... San Juan: ASCE, 2016. 1050-1060. doi:https://doi.org/10.1061/9780784479827.106.

EL DEBS, L. C; FERREIRA, S. L. Proposta de ferramenta computacional com conceito BIM para elaboração de projetos de painéis pré-fabricados de fachada. In: ENCONTRO NACIONAL DE TECNOLOGIA DO AMBIENTE CONSTRUÍDO, 14., 2012, Juiz de Fora. Anais... Juiz de Fora: ANTAC, 2012. 1441-1446.

HAMDAN, Samer Bu et al. A BIM-based simulation model for inventory management in panelized construction. In: INTERNATIONAL SYMPOSIUM ON AUTOMATION AND ROBOTICS IN CONSTRUCTION AND MINING (ISARC), 32., 2015, Oulu, Finlândia. Proceedings... [S.I. : s.n.]. 312-317. doi:https://doi.org/10.22260/ISARC2015/0092.

INYANG, Ndukeabasi et al. A VR model of ergonomics and productivity assessment in panelized construction production line. In: CONSTRUCTION RESEARCH CONGRESS, 2012, West Lafayette, Estados Unidos. Proceedings... West Lafayette: ASCE, 2012. 1084-1093. doi:https://doi.org/10.1061/9780784412329.109.

LIU, H. et al. Automated production planning in panelized construction enabled by integrating discrete-event simulation and BIM. In: THE CADADIAN SOCIETY OF CIVIL ENGINEERS $5^{\text {th }}$ INTERNATIONAL/ $11^{\text {th }}$ CONSTRUCTION SPEACIALTY CONFERENCE, 2015, Vancouver, Canada. Proceedings... Vancouver: University of British Columbia, 2015. 1-10.

LIU, Hexu et al. An automatic scheduling approach: building information modeling-based onsite scheduling for panelized construction. In: CONSTRUCTION RESEARCH CONGRESS, 2014, Atlanta, Estados Unidos. Proceedings... Atlanta: ASCE, 2014. p. 1666-1675. doi:https://doi.org/10.1061/9780784413517.170.

LIU, Hexu. BIM-based automated planning for panelized construction in the light-frame building industry. 2016. 207f. Tese (Doutorado em Construction Engineering and Management) - Department of Civil and Enviromental Engineering, University of Alberta, Edmonton, Canadá. 2016. doi:https://doi.org/10.7939/R39G5GQ7Z.

LIU, Hexu; AL-HUSSEIN, Mohamed; LU, Ming. BIM-based integrated approach for detailed construction scheduling under resource constraints. Automation in Construction, v. 53, p. 29-43, 2015. doi:https://doi.org/10.1016/j.autcon.2015.03.008.

LU, Na; KORMAN, Thomas. Implementation of building information modeling (BIM) in modular construction: benefits and challenges. In: CONSTRUCTION RESEARCH CONGRESS, 2010, Banff, Canada. Proceedings... Banff: ASCE, 2010. 11361145. doi:https://doi.org/10.1061/41109(373)114.

MUÑOZ, Susana Inés Segura et al. Revisão sistemática de literatura e metanálise: noções básicas sobre seu desenho, interpretação e aplicação na área da saúde. In: BRAZILIAN NURSING COMMUNICATION SYMPOSIUM, 8., 2002, São Paulo, Brasil. Proceedings... Escola de Enfermagem de Ribeirão Preto - USP. Disponível em: http://www.proceedings.scielo.br/scielo.php?script=sci_arttext\&pid=MSC0000000052002000200010\&lng=en\&nrm=abn. Acesso em: 16/08/2017.

PEREIRA, Ângela Lima; BACHION, Maria Márcia. Atualidades em revisão sistemática de literatura, critérios de força e grau de recomendação de evidência. Revista Gaúcha de Enfermagem, v. 27, n. 4, p. 491, 2006.

RAJENDRAN, S.; CLARKE, B. Building information modeling: safety benefits \& opportunities. Professional Safety, 2011. Disponível em: <https://www.onepetro.org/journal-paper/ASSE-11-10-44>. Acesso em: 19/8/2017.

SAID, Hisham M.; CHALASANI, Tejaswini; LOGAN, Stephanie. Exterior prefabricated panelized walls platform optimization. Automation in Construction, v. 76, p. 1-13, 2017. doi:https://doi.org/10.1016/j.autcon.2017.01.002.

SALAMA, Tarek; SALAH, Ahmad; MOSELHI, Osama. Alternative scheduling and planning processes for hybrid offsite construction. In: INTERNATIONAL SYMPOSIUM ON AUTOMATION AND ROBOTICS IN CONSTRUCTION AND MINING (ISARC), 33., 2016, Auburn, Estados Unidos. Proceedings... [S.I : s.n]. 165-173. doi:https://doi.org/10.22260/ISARC2016/0021.

SALZER, C.; WALLBAUM, H.; LOPEZ, L. F.; KOUYOUMJI, J. L. Sustainability of social housing in asia: a holistic multiperspective development process for bamboo-based construction in the philippines. Sustainability, v. 8, n. 2, 2016. MDPI AG. Disponível em: <https://doaj.org/article/8971d8cf832d4ff097cb6f63872cf528>. Acesso em: 19/08/2017. 
SAMARASINGHE, T. et al. BIM software framework for prefabricated construction: case study demonstrating BIM implementation on a modular house. In: INTERNATIONAL CONFERENCE ON STRUCTURAL ENGINEERING AND CONSTRUCTION MANAGEMENT, 6., 2015, Kandy, Sri Lanka. Proceedings... [S.I : s.n]. 154-162.

SAMPAIO, R. F; MANCINI, M. C. Estudos de revisão sistemática: um guia para síntese criteriosa da evidência científica. Revista Brasileira de Fisioterapia, v. 11, n. 1, p. 83-89, jan./fev. 2007. doi:http://dx.doi.org/10.1590/S1413-35552007000100013.

TAM, R. An analysis of growth opportunities of pre-fabricated housing in Vietnam. 2013. 27 f.. Trabalho Final de Curso (Bachelor of Science in Wood Products Processing em Administração) - Faculty Forestry, University of British Columbia , Vancouver, 2013. . Disponível em: 〈https://open.library.ubc.ca/cIRcle/collections/undergraduateresearch/52966/items/1.0103151>. Acesso em: $2 / 8 / 2017$

VIVAN, André Luiz; PALIARI, José Carlos; NOVAES, Celso Carlos. Vantagem Produtiva do Sistema Light Steel Framing: da construção enxuta à racionalização construtiva. In: ENCONTRO NACIONAL DE TECNOLOGIA DO AMBIENTE CONSTRUídO, 13., 2010, Canela. Anais... Canela: ANTAC, 2010.

\section{${ }^{1}$ Pedro Henrique Pinto Bezerra}

Mestrando. Endereço postal: Rua Visconde de Nácar, 359, Curitiba, PR, Brasil, CEP: 80410-200

\section{Adriana de Paula Lacerda Santos}

Doutora. Endereço postal: Universidade Federal do Paraná, Setor de Tecnologia, Centro Politécnico, s/n, Curitiba, PR, Brasil, CEP: 81531-990

\section{${ }^{3}$ Sergio Scheer}

Doutor. Endereço postal: Centro de Estudos de Engenharia Civil, Centro Politécnico, UFPR, Av. Francisco H. dos Santos, s/n, Curitiba, PR, Brasil, CEP: 81531-980 